\title{
Toxidez de zinco no crescimento e nutrição de Eucalyptus maculata e Eucalyptus urophylla em solução nutritiva ${ }^{(1)}$
}

\author{
Cláudio Roberto Fonsêca Sousa Soares ${ }^{(2)}$, Paulo Henrique Grazziotti(3), José Oswaldo Siqueira ${ }^{(3)}$, \\ Janice Guedes de Carvalho(3) e Fátima Maria Souza Moreira ${ }^{(3)}$
}

\begin{abstract}
Resumo - O objetivo do trabalho foi avaliar o efeito de doses excessivas de Zn no crescimento e nutrição do eucalipto, em casa de vegetação. Mudas de Eucalyptus maculata e Eucalyptus urophylla foram crescidas em vasos contendo $2 \mathrm{~L}$ de solução nutritiva de Clark, adicionando-se doses crescentes de $\mathrm{Zn}:$ 0, 400, 800, 1.200 e $1.600 \mu \mathrm{M}$ fornecidas como $\mathrm{ZnSO}_{4}$. Após cinco semanas, as plantas exibiram clorose internerval, escurecimento das raízes e inibição do crescimento, mesmo nas doses mais baixas do metal. A dose crítica de Zn na solução (para redução de $10 \%$ na matéria seca da parte aérea) em E. maculata foi de 170,3 $\mu \mathrm{M}$, e em E. urophylla, 73,0 $\mu \mathrm{M}$. Os níveis críticos de toxidez de Zn na planta foram de $853 \mathrm{mg} \mathrm{kg}^{-1} \mathrm{em} \mathrm{E}$. maculata, e $697,8 \mathrm{mg} \mathrm{kg}^{-1} \mathrm{em} \mathrm{E}$. urophylla. Esses resultados indicam que E. maculata é mais tolerante ao Zn do que E. urophylla. Altas doses de Zn reduziram as concentrações de $\mathrm{Fe}$ e $\mathrm{Ca}$ na matéria seca da parte aérea a níveis considerados deficientes para o crescimento das duas espécies. A translocação de Fe das raízes para a parte aérea foi reduzida, independentemente da espécie, de $21 \%$ no controle para apenas $2 \%$ em $1.600 \mu \mathrm{M}$ de $\mathrm{Zn}$, indicando forte relação entre a queda na produção de matéria seca e a ocorrência da deficiência induzida de Fe nas plantas.
\end{abstract}

Termos para indexação: eucalipto, nutrição das plantas, oligoelementos, metais pesados.

\section{Zinc toxicity on growth and nutrition of Eucalyptus maculata and Eucalyptus urophylla in nutrient solution}

\begin{abstract}
The objective of this work was to evaluate the effect of excessive doses of Zn on growth and nutrition of eucalyptus in a greenhouse experiment. Seedlings of Eucalyptus maculata and Eucalyptus urophylla were grown in pots containing $2 \mathrm{~L}$ of Clark's nutrient solution, adding increasing levels of $\mathrm{Zn}$, in $\mu \mathrm{M}$ : 0, 400, 800, 1,200 and 1,600 supplied as $\mathrm{ZnSO}_{4}$. After five weeks, plants exhibited internerval chlorosis, dark roots and growth inhibition, even in lower metal levels. The critical metal dose in the solution, for $10 \%$ reduction of shoot dry matter, was $170.3 \mu \mathrm{M}$ and $73.0 \mu \mathrm{M}$ in E. maculata and E. urophylla, respectively. The plant critical toxicity levels were $853 \mathrm{mg} \mathrm{kg}^{-1}$ in E. maculata and $697.8 \mathrm{mg} \mathrm{kg}^{-1}$ in E. urophylla. These results indicate that E. maculata is more tolerant to $\mathrm{Zn}$ than E. urophylla. High levels of $\mathrm{Zn}$ reduced concentrations of $\mathrm{Fe}$ and $\mathrm{Ca}$ in shoot dry matter to levels considered deficient for the growth of both species. Translocation of Fe from roots to shoots was also reduced, independently of the species, of $21 \%$ in control to $2 \%$ in $1,600 \mu \mathrm{M} \mathrm{Zn}$, indicating strong relationship among dry matter reduction and induced Fe-deficiency in plants.
\end{abstract}

Index terms: eucalyptus, plant nutrition, trace elements, heavy metals.

(1) Aceito para publicação em 9 de maio de 2000. Extraído da dissertação de mestrado, apresentada pelo primeiro autor à Universidade Federal de Lavras (UFLA), Lavras, MG. Trabalho financiado pelo convênio Cia. Mineira de Metais/FAEPE e FAPEMIG.

(2) UFLA, Dep. de Ciência do Solo, Caixa Postal 37, CEP 37200-000 Lavras, MG. Bolsista da CAPES. E-mail: crfsousa@ufla.br

(3) UFLA, Dep. de Ciência do Solo. Bolsista do CNPq. E-mail: grazziot@ufla.br, siqueira@ufla.br, janicegc@ufla.br, fmoreira@ufla.br

\section{Introdução}

O aumento de atividades industriais e de mineração e a crescente necessidade de aplicação de biossólidos e fertilizantes no solo têm alterado o ciclo geoquímico e fluxo natural dos metais pesados, aumentando a liberação desses elementos na biosfera (Kabata-Pendias \& Pendias, 1985). Com isso, a contaminação do solo, cursos de água e lençol freático 
são problemas ambientais graves que necessitam de efetiva solução tecnológica (Salt et al., 1995). Entre as inúmeras estratégias de remediação, a fitorremediação é uma alternativa promissora, destacando-se a introdução de maciços florestais em áreas contaminadas com metais pesados. Estudos básicos sobre fitotoxicidade dos metais pesados e da tolerância das espécies aos estresses impostos pelo excesso destes são essenciais para o desenvolvimento de tecnologia para fitorremediação. Conhecer os padrões de absorção, translocação e acúmulo de íons metálicos, os limites de tolerância e os sintomas de fitotoxidez das espécies com potencial para introdução em áreas contaminadas, tornou-se de grande importância acadêmica e tecnológica (Huang \& Cunningham, 1996).

Apesar de o Zn ser um micronutriente essencial, este elemento pode afetar o crescimento e metabolismo normal de espécies vegetais, quando presente em níveis tóxicos no ambiente (Marschner, 1995). De maneira geral, os sintomas de toxidez de $\mathrm{Zn}$ em plantas são caracterizados por redução no crescimento e clorose de folhas, semelhantemente à deficiência de Fe (Fontes \& Cox, 1998a). Não se sabe a causa da deficiência induzida de Fe provocada pelo excesso de $\mathrm{Zn}$, mas a similaridade entre o raio iônico desses elementos tem sido apontada como mecanismo deste fenômeno (Woolhouse, 1983). Os níveis críticos de toxidez de $\mathrm{Zn}$ em solução nutritiva e no tecido vegetal têm sido determinados em estudos com espécies herbáceas acumuladoras de metais (Shaw, 1989; Pollard \& Baker, 1997), algumas espécies arbóreas de clima temperado (Baker, 1981) e arbóreas dos trópicos (Marques et al., 2000). No entanto, sobre o eucalipto, são encontrados na literatura apenas trabalhos relacionados à deficiência nutricional de micronutrientes (Dell \& Robinson, 1993; Dell et al., 1995), e existe carência de estudos que tratem do efeito isolado da toxidez por metais pesados, e carência, também, de determinações de níveis críticos de toxidez desses elementos.

O objetivo deste trabalho foi avaliar o efeito de doses excessivas de $\mathrm{Zn}$ no crescimento e nutrição mineral de Eucalyptus maculata e Eucalyptus urophylla em solução nutritiva.

\section{Material e Métodos}

O ensaio foi desenvolvido em casa de vegetação do Departamento de Ciência do Solo da Universidade Federal de Lavras, no período de junho a agosto de 1996. Mudas de E. maculata e E. urophylla foram produzidas em substrato contendo areia + vermiculita $(50 \% \mathrm{v} / \mathrm{v})$, irrigadas semanalmente com solução nutritiva de Clark (1975) preparada com reagentes pa, e que continha $7,26 \mathrm{mM}$ de $\mathrm{N}-\mathrm{NO}_{3}{ }_{3} ; 0,90 \mathrm{mM}$ de N-NH${ }_{4}^{+} ; 0,07 \mathrm{mM}$ de $\mathrm{P} ; 1,80 \mathrm{mM}$ de $\mathrm{K} ; 2,60 \mathrm{mM}$ de $\mathrm{Ca} ; 0,60 \mathrm{mM}$ de $\mathrm{Mg} ; 0,50 \mathrm{mM}$ de $\mathrm{S}$; 7,0 $\mu \mathrm{M}$ de $\mathrm{Mn} ; 2 \mu \mathrm{M}$ de $\mathrm{Zn} ; 0,5 \mu \mathrm{M}$ de $\mathrm{Cu} ; 19 \mu \mathrm{M}$ de B; $0,60 \mu \mathrm{M}$ de Mo e $38 \mu \mathrm{M}$ de Fe complexado com $\mathrm{Na}_{2}$-EDTA; nesta etapa foi utilizada solução meia-força. Duas semanas após a emergência do primeiro par de folhas, as mudas foram transplantadas para bandejas coletivas com capacidade para $30 \mathrm{~L}$, contendo a mesma solução nutritiva de Clark meia-força, com aeração constante e pH mantido em 5,5 pela adição de $\mathrm{NaOH}$ ou $\mathrm{HCl} 0,1 \mathrm{~N}$, onde permaneceram durante 15 dias. Em seguida, esta solução foi substituída pela solução nutritiva de Clark (1975), permanecendo nesta por mais 15 dias, quando as mudas atingiram altura média de $20 \mathrm{~cm}$. Após padronização quanto à altura e vigor, transplantaram-se duas plantas para cada um dos vasos de $2 \mathrm{~L}$; esses vasos constituíram a parcela experimental, e foram dispostos em esquema fatorial 2x5: duas espécies de eucalipto e cinco doses $(0,400,800,1.200$ e $1.600 \mu \mathrm{M}$ de $\mathrm{Zn}$ fornecido como $\mathrm{ZnSO}_{4}$ ), em delineamento formado por blocos casualizados, com quatro repetições. A solução nutritiva contendo a respectiva dose de $\mathrm{Zn}$ foi renovada semanalmente, e o pH foi mantido em 5,5 pela adição de $\mathrm{NaOH}$ ou de $\mathrm{HCl} 0,1 \mathrm{~N}$.

Após cinco semanas de exposição ao $\mathrm{Zn}$ nestas condições, procedeu-se à colheita das plantas, dividindo-as em parte aérea e raízes. Após lavadas, as partes das plantas foram secadas em estufa com circulação de ar a $65^{\circ} \mathrm{C}$ até peso constante, pesadas, e moídas em moinho tipo Willey equipado com peneira com malha de $0,38 \mathrm{~mm}$. Em seguida, efetuou-se a digestão nítrico-perclórica do material seco e moído, obtendo-se extratos para determinação dos teores de $\mathrm{P}$ pelo método colorimétrico, $\mathrm{K}$ por fotometria de chama, Ca e Mg por espectrofotometria de absorção atômica. Os teores de $\mathrm{Zn}, \mathrm{Cu}, \mathrm{Mn}$ e Fe também foram determinados por espectrofotometria de absorção atômica.

$\mathrm{O}$ acúmulo de $\mathrm{P}, \mathrm{K}, \mathrm{S}, \mathrm{Ca}, \mathrm{Mg}, \mathrm{Zn}, \mathrm{Cu}, \mathrm{Mn}$ e Fe na parte aérea e nas raízes foi calculado com base nas concentrações e produção de matéria seca. Mediante o acúmulo de nutrientes e metais pesados, calcularam-se os índices de translocação (IT) desses elementos de acordo com Abichequer \& Bohnen (1998), com o uso da seguinte equação: 
IT = (Quantidade acumulada na parte aérea/quantidade acumulada na planta) x 100 .

Os resultados foram submetidos a análise de variância por meio do programa estatístico SAEG (Euclydes, 1983). As equações de regressão foram escolhidas pelo melhor ajuste através do programa Table Curve 2D for Windows v. 2.03 (Jandel Corporation), e o valor de $\mathrm{F}$ das regressões foi corrigido pelo programa Fcalc32 for Windows v. 1.1 (Moura Filho \& Cruz, 1996). As doses críticas de toxidez de Zn na solução para redução da produção de parte aérea e raízes (concentração do elemento na solução nutritiva que reduz em $10 \%$ os parâmetros de crescimento) e os níveis críticos de toxidez na planta (concentração foliar de $\mathrm{Zn}$ requerida para inibir em $10 \%$ a matéria seca da parte aérea) foram estimados com base nas equações de regressão obtidas em relação a cada espécie em resposta às doses do elemento.

\section{Resultados e Discussão}

No início da aplicação dos tratamentos, houve presença de clorose internerval nas folhas mais novas de ambas as espécies, sintoma típico de deficiência induzida de Fe. Para Breckle \& Kahle (1992), a ocorrência de clorose pode estar relacionada com a deficiência múltipla de vários elementos que participam da formação, multiplicação e funcionamento de cloroplastos, ou na síntese de clorofila. Essa deficiência de nutrientes pode ser resultado da inibição competitiva que o $\mathrm{Zn}$ pode exercer na absorção de outros íons (Malavolta, 1994). Em E. maculata, as doses de 1.200 e $1.600 \mu \mathrm{M}$ de $\mathrm{Zn}$ causaram morte das plantas, deixando-as com as folhas esverdeadas e aspecto coriáceo. Isto se deve, possivelmente, ao efeito do Zn na extensibilidade e/ou síntese de material de parede celular, conforme discutido em Barceló \& Poschenrieder (1992). Em E. urophylla, verificouse o aparecimento de pontuações arroxeadas próximas às nervuras das folhas basais e à curvatura vertical destas, sintomas, estes, semelhantes aos observados em Glycine max L. sob toxidez de Zn (Fontes \& Cox, 1998b). Raízes escurecidas e pouco desenvolvidas foram os sintomas observados em ambas as espécies, mesmo na dose mais baixa de $\mathrm{Zn}$ em solução $(400 \mu \mathrm{M})$. De acordo com Baccouch et al. (1998), este tipo de alteração pode ser conseqüência da deficiência induzida de Ca.
O aumento das doses de $\mathrm{Zn}$ na solução nutritiva exerceu efeito negativo acentuado sobre o crescimento das plantas. A matéria seca da parte aérea (PA) e das raízes $(\mathrm{R})$ e a razão $\mathrm{R} / \mathrm{PA}$ diminuíram com o aumento das doses de $\mathrm{Zn}$ em solução, e não ocorreu efeito significativo nas espécies, em nenhuma dessas variáveis (Figura 1). A dose $1.600 \mu \mathrm{M}$ de Zn causou redução de $79 \%$ e $97 \%$ na matéria seca da parte aérea e raízes, respectivamente, com conseqüente redução da razão R/PA, que reduziu de 0,38 no controle para $0,14 \mathrm{em} 1.600 \mu \mathrm{M}$ de $\mathrm{Zn}$, indicando, assim, que o elemento promove maior dano na produção de massa de raízes do que da parte aérea.

As doses críticas de toxidez de $\mathrm{Zn}$ na solução (DCT) da matéria seca da parte aérea e das raízes apresentaram comportamento diferenciado entre as espécies (Tabela 1). A DCT foi 2,33 vezes maior em E. maculata, indicando, assim, que essa espécie tolera melhor o $\mathrm{Zn}$ quando em doses moderadas. A DCT de E. maculata foi superior aos $140 \mu \mathrm{M}$ de Zn encontrados por Davis et al. (1978) em Hordeum vulgare L., o que indica a maior tolerância do E. maculata em relação a esta espécie. A dose requerida para inibir a matéria seca da parte aérea em $50 \%\left(\mathrm{DT}_{50}\right)$ foi de 4,04 e 6,68 vezes maior que a DCT em E. maculata e E. urophylla, respectivamente, o que indica também a maior tolerância de E. maculata. A DCT e a DT 50 de Zn da matéria seca de raízes foram semelhantes entre as espécies, e inferiores às observadas na matéria seca da parte aérea, reafirmando, assim, o maior efeito tóxico desse metal nas raízes.

A concentração de $\mathrm{Zn}$ na matéria seca da parte aérea foi maior em E. urophylla, atingindo o máximo de $1.194 \mathrm{mg} \mathrm{kg}^{-1}$ na dose de $634 \mu \mathrm{M}$, enquanto em E. maculata a concentração máxima foi de $1.033 \mathrm{mg} \mathrm{kg}^{-1}$, obtida na dose de $537 \mu \mathrm{M}$ (Figura 2). Em comparação ao controle, a dose de $400 \mu \mathrm{M}$ de Zn elevou em aproximadamente 200 vezes a concentração desse elemento na parte aérea de E. urophylla, e em 11 vezes na de E. maculata. O nível crítico de toxidez (NCT) de Zn na parte aérea de E. maculata foi de $853 \mathrm{mg} \mathrm{kg}^{-1}$, portanto superior ao do E. urophylla, $697,8 \mathrm{mg} \mathrm{kg}^{-1}$, indicando maior susceptibilidade ao $\mathrm{Zn}$ desta última em relação à primeira. Os NCT deste elemento nas mudas de eucalipto apresentam-se muito superiores aos da 

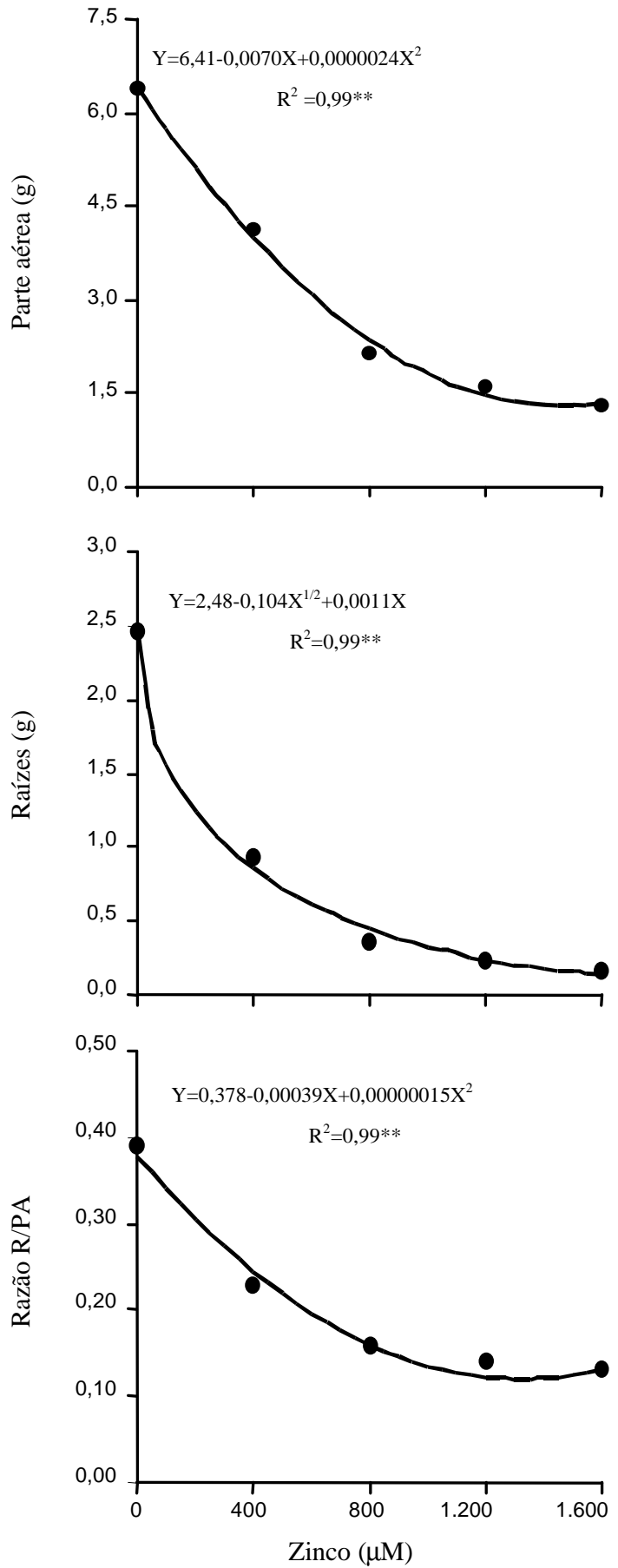

Figura 1. Matéria seca da parte aérea $(P A)$ e raízes $(R)$ e razão R/PA em mudas de eucalipto em resposta a doses de Zn em solução nutritiva, em média das espécies E. maculata e E. urophylla $(* * \mathrm{p} \leq 0,01)$. faixa de 100 a $400 \mathrm{mg} \mathrm{kg}^{-1}$, considerada tóxica para o crescimento de várias espécies (Kabata-Pendias \& Pendias, 1985). Isto indica tolerância elevada destas espécies de eucalipto ao $\mathrm{Zn}$.

A concentração de $\mathrm{Zn}$ na matéria seca das raízes aumentou progressivamente com a elevação das doses aplicadas, verificando-se concentração de $333,73 \mathrm{mg} \mathrm{kg}^{-1}$ no controle, e de $6.740 \mathrm{mg} \mathrm{kg}^{-1} \mathrm{em}$ $1.600 \mu \mathrm{M}$ de $\mathrm{Zn}$. Isto mostra relação positiva entre a concentração de $\mathrm{Zn}$ nas raízes e a capacidade de absorção desse elemento da solução. Houve aumento na concentração de $\mathrm{Cu}$ na matéria seca da parte aérea de E. urophylla, e as doses de Zn não tiveram efeito na sua concentração em E. maculata. Ao contrário da parte aérea, a concentração de $\mathrm{Cu}$ nas raízes de E. urophylla não foi influenciada pelas doses de $\mathrm{Zn}$, enquanto em $E$. maculata houve efeito, mas não foi encontrado ajuste polinomial.

A dose mais baixa de $\mathrm{Zn}$ em solução $(400 \mu \mathrm{M})$ foi suficiente para reduzir em $57 \%$ a concentração de Mn na matéria seca da parte aérea das espécies, porém não atingiu a faixa considerada de deficiência, que é de 12 a $15 \mathrm{mg} \mathrm{kg}^{-1}$ em E. maculata (Dell \& Robinson, 1993) e de 5 a $16 \mathrm{mg} \mathrm{kg}^{-1}$ em E. urophylla (Dell et al., 1995). A concentração de Mn nas raízes também reduziu drasticamente já na primeira dose de $\mathrm{Zn}(400 \mu \mathrm{M})$, em que ocorreu decréscimo de $68 \%$ em E. maculata e $51 \%$ em E. urophylla, ficando evidente a inibição competitiva entre esses dois elementos. A concentração de Fe na parte aérea das espécies foi altamente reduzida com o aumento das doses de $\mathrm{Zn}$ em solução, observando-se redução de $65 \%$ na dose de $1.600 \mu \mathrm{M}$. A concentração de Fe na parte aérea de E. urophylla está abaixo da faixa de concentração de 52 a

Tabela 1. Doses críticas de toxidez de Zn $(\mu \mathrm{M})$ em solução nutritiva para duas espécies de eucalipto.

\begin{tabular}{lccccc}
\hline \multirow{2}{*}{$\begin{array}{c}\text { Doses } \\
\text { críticas }\end{array}$} & \multicolumn{2}{c}{ Eucalyptus } & maculata & & \multicolumn{2}{c}{ Eucalyptus urophylla } \\
\cline { 2 - 3 } \cline { 5 - 6 } & Parte aérea & Raízes & & Parte aérea & Raízes \\
\hline $\mathrm{DCT}^{(1)}$ & 170,3 & 40,3 & & 73,0 & 41,4 \\
$\mathrm{DT}_{50}{ }^{(2)}$ & 687,8 & 277,5 & & 487,5 & 273,9 \\
\hline
\end{tabular}

(1) Concentração na solução requerida para inibir em $10 \%$ o parâmetro de crescimento. (2) Concentração na solução requerida para inibir em $50 \%$ o parâmetro de crescimento. 
Parte aérea
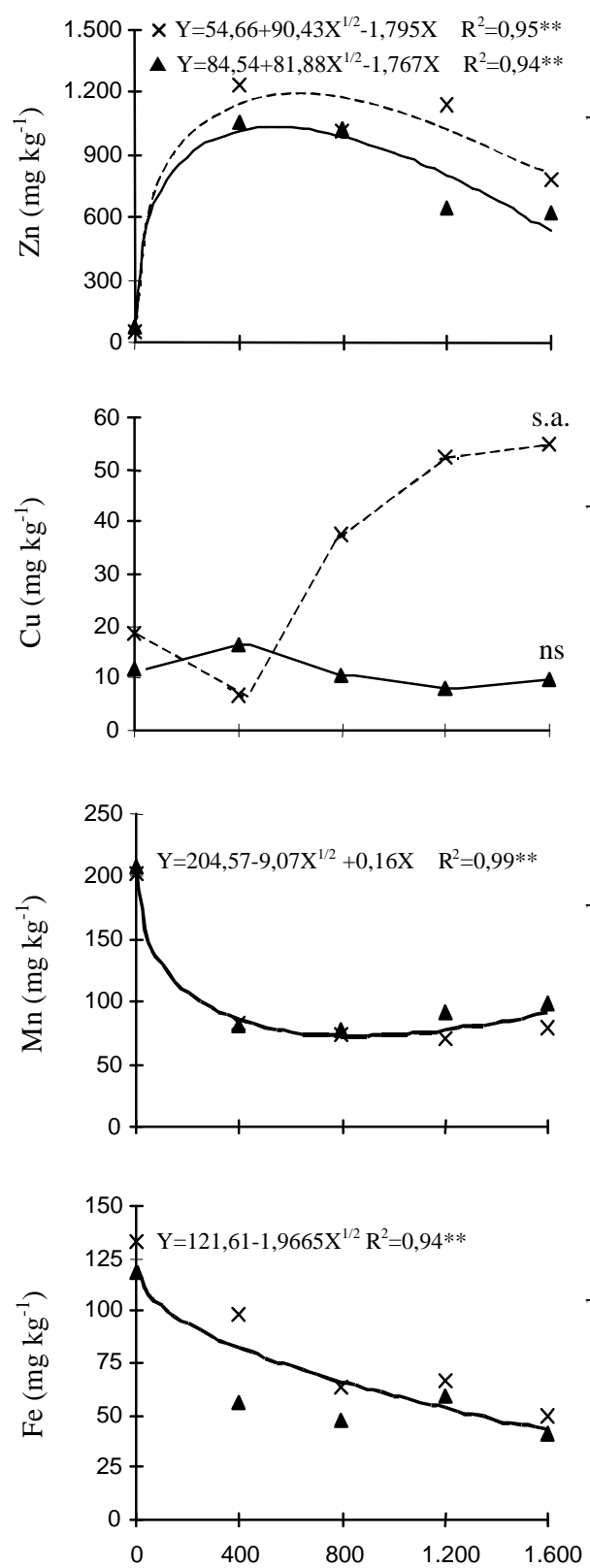

Zinco $(\mu \mathrm{M})$
Raiz
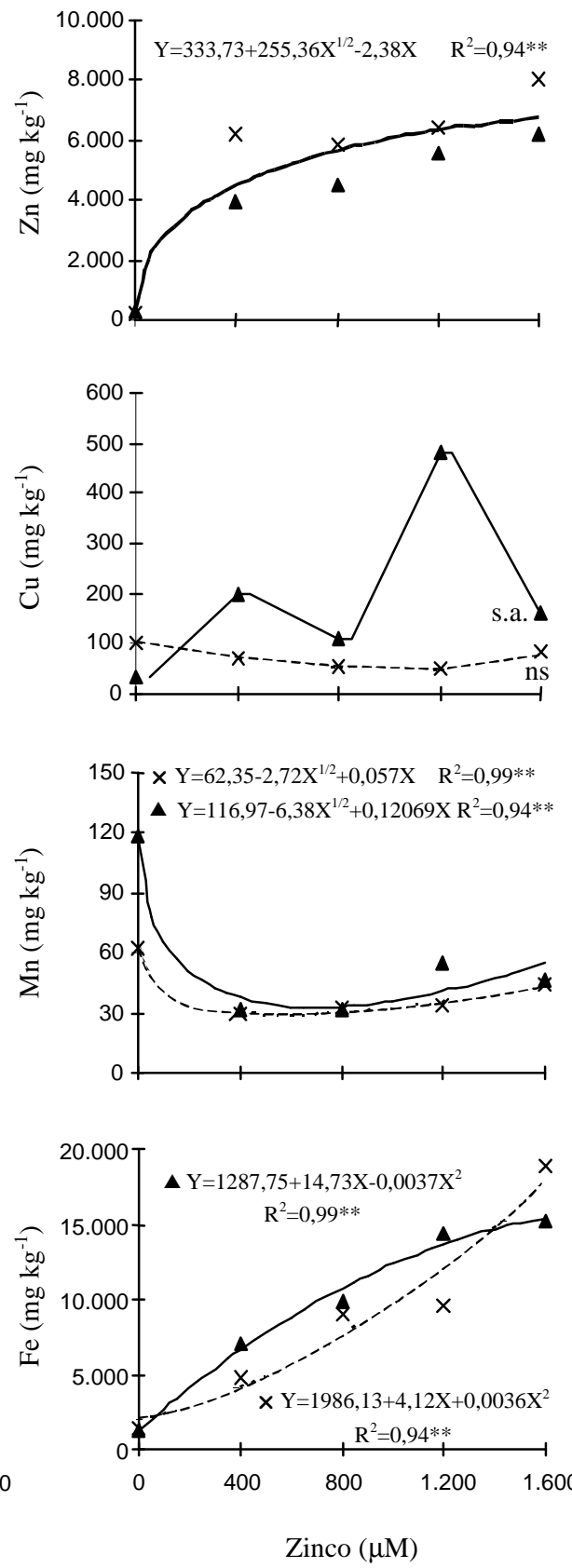

Média

Figura 2. Concentração de $\mathrm{Zn}, \mathrm{Cu}, \mathrm{Mn}$ e $\mathrm{Fe}$ na matéria seca da parte aérea e raiz em mudas de eucalipto (MAC: E. maculata; URO: E. urophylla) em resposta a doses de Zn em solução nutritiva (ns: não-significativo; s.a.: sem ajuste polinomial; $* \mathrm{p} \leq 0,05 ; * * \mathrm{p} \leq 0,01$ ). 
$97 \mathrm{mg} \mathrm{kg}^{-1}$, considerada adequada para o crescimento dessa espécie (Dell et al., 1995). Segundo Woolhouse (1983), o antagonismo entre Zn e Fe é resultante da semelhança dos raios iônicos desses elementos $(0,083 \mathrm{~nm})$. Em Glycine $\max \mathrm{L}$., a maior e menor produção da parte aérea, em resposta à toxidez de $\mathrm{Zn}$, foi coincidente com a maior e menor concentração de Fe (Fontes \& Cox, 1998a), tal como verificado na parte aérea do eucalipto. Isto sugere que a deficiência de Fe é um dos fatores que prejudica o crescimento das plantas expostas à toxidez de $\mathrm{Zn}$, mas não é necessariamente a causa. As espécies de eucalipto apresentaram comportamento diferenciado em relação à concentração de Fe nas raízes, ocorrendo aumento da concentração desse elemento com a elevação das doses de $\mathrm{Zn}$. Isto mostra que elevadas doses de $\mathrm{Zn}$ não afetam a absorção de $\mathrm{Fe}$, e sim, a translocação do Fe absorvido para a parte aérea. Portanto, a elevação nas doses de $\mathrm{Zn}$ reduziram marcadamente a concentração de Mn e Fe nas espécies de eucalipto.

As doses de $\mathrm{Zn}$ influenciaram de maneira diferente a translocação de $\mathrm{Zn}$ e Fe, porém não foram encontrados ajustes polinomiais nesta resposta (Figura 3). O IT do Zn não apresentou comportamento diferenciado entre as espécies com valores superiores a $40 \%$, o que indica a sua alta capacidade de translocar-se das raízes para a parte aérea do eucalipto. Os valores de IT para este metal elevaramse até a dose de $800 \mu \mathrm{M}$, reduzindo-se a partir desta. A translocação do $\mathrm{Fe}$ reduziu-se de $21 \%$, na dose $0 \mu \mathrm{M}$, para $7 \%$ na dose $400 \mu \mathrm{M}$, e apenas $2 \%$ com $1.600 \mu \mathrm{M}$ de $\mathrm{Zn}$, independentemente da espécie. Isto evidencia o forte antagonismo entre $\mathrm{Zn}$ e Fe nestas espécies de eucalipto. Esses resultados corroboram os sintomas de clorose internerval observados, os quais são típicos de deficiência de Fe. Agarwala et al. (1977) também observaram que o excesso de $\mathrm{Zn}^{2+}$ reduz a translocação de Fe em cevada, sem, entretanto, isto estar relacionado com a ação induzida do $\mathrm{Zn}$. É sabido que o excesso de $\mathrm{Zn}$ pode interferir no metabolismo de carboidratos, inibindo o transporte de fotoassimilados (Samarakoon \& Rauser, 1979), e afetando o crescimento da planta.

A concentração de nutrientes nas diferentes partes das plantas foi influenciada $(\mathrm{p} \leq 0,01)$ pelas doses de $\mathrm{Zn}$ em solução, exceto o K na parte aérea (Figu- ra 4). E. maculata e E. urophylla apresentaram, respectivamente, decréscimo de até $84 \%$ e $60 \%$, na concentração de $\mathrm{K}$ nas raízes, caracterizando o processo de inibição no processo de absorção. Verificou-se aumento da concentração de $\mathrm{P}$ na parte aérea, atingindo-se o máximo de $3,0 \mathrm{~g} \mathrm{~kg}^{-1}$ na dose $820 \mu \mathrm{M}$ de $\mathrm{Zn}$, o que corresponde a um aumento de $82 \% \mathrm{em}$ relação ao tratamento sem $\mathrm{Zn}$ adicional. Nas raízes, ocorreu comportamento diferenciado entre as espé-

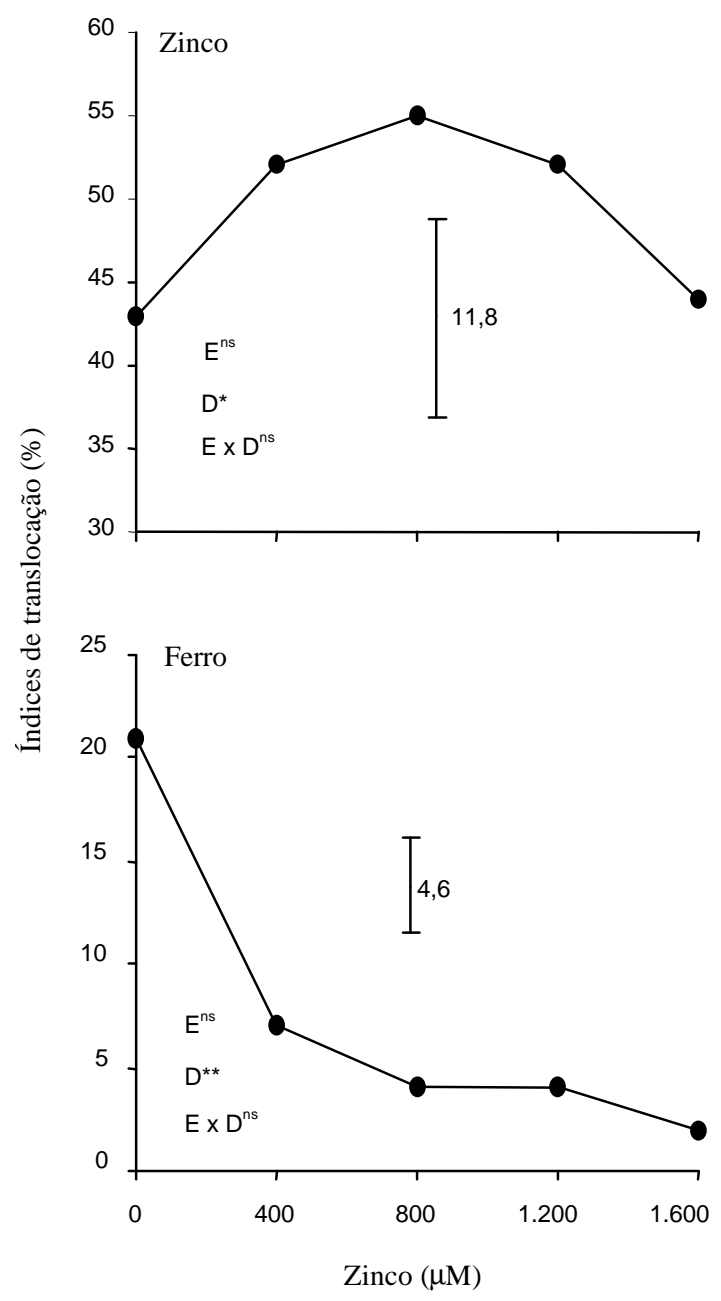

Figura 3. Índices de translocação (\%) de Zn e Fe em duas espécies de eucalipto crescidas em solução nutritiva com doses crescentes de Zn. E (espécie), D (dose de Zn) e interação Ex D (ns: não-significativo; *p $\leq 0,05$; $* * \mathrm{p} \leq 0,01)$. A barra vertical representa a DMS (dose) do teste de Tukey a $5 \%$ de probabilidade. 

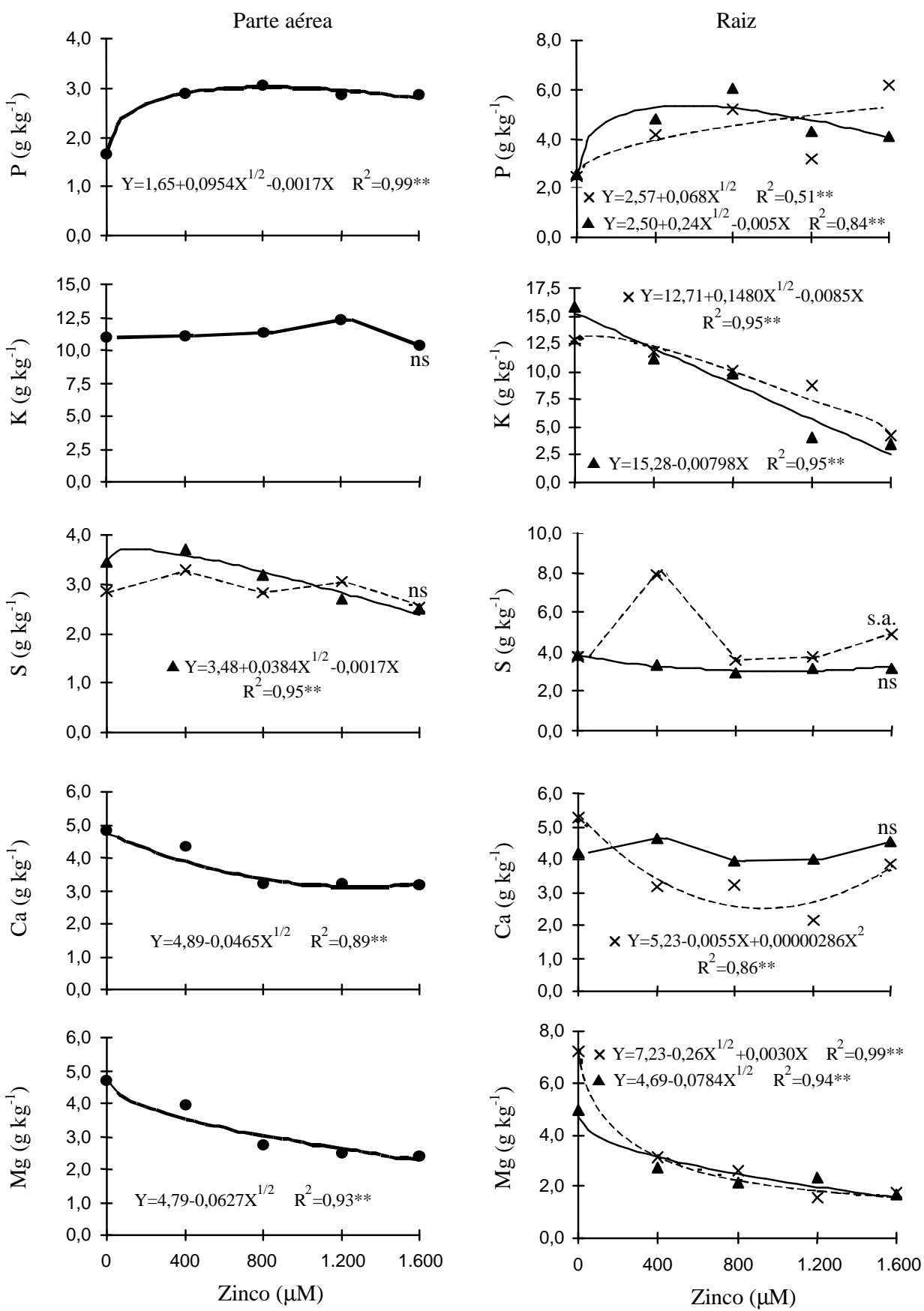

$$
\text { ---x--- URO M MAC Média }
$$

Figura 4. Concentração de $\mathrm{P}, \mathrm{K}, \mathrm{S}, \mathrm{Ca}$ e $\mathrm{Mg}$ na matéria seca da parte aérea e raiz em mudas de eucalipto (MAC: E. maculata; URO: E. urophylla) em resposta a doses de Zn em solução nutritiva (ns: não-significativo; s.a.: sem ajuste polinomial; **p $\leq 0,01$ ). 
cies, com E. maculata apresentando resposta do tipo raiz quadrática, verificando-se elevação na concentração de $\mathrm{P}$ nas doses mais baixas de $\mathrm{Zn}$. O aumento da concentração de $\mathrm{P}$ nos tecidos deve-se, possivelmente, ao efeito de concentração, uma vez que se observou acentuada redução na produção de matéria seca (Figura 1). A concentração de $S$ nos tecidos foi pouco influenciada pelas doses de $\mathrm{Zn}$ em solução.

Não houve comportamento diferenciado entre as espécies para a concentração de Ca na parte aérea. Ocorreu decréscimo de $38 \%$ na dose de $1.600 \mu \mathrm{M}$ de $\mathrm{Zn}$, quando a concentração mínima de $3,0 \mathrm{~g} \mathrm{~kg}^{-1}$ de Ca foi obtida, a qual encontra-se abaixo da faixa adequada de 3,1 a 4,3 $\mathrm{g} \mathrm{kg}^{-1}$ em relação ao crescimento de E. urophylla (Dell et al., 1995). Resposta quadrática foi obtida na concentração de Ca nas raízes de E. urophylla, observando-se decréscimo nas doses intermediárias de Zn, enquanto em $E$. maculata não foi afetada significativamente pelas doses de $\mathrm{Zn}$ em solução. Brown et al. (1995) também observaram redução na concentração de Ca nas raízes de Thlaspi caerulescens em dose de $3.160 \mu \mathrm{M}$ de $\mathrm{Zn}$. A concentração de $\mathrm{Mg}$ nos tecidos também foi influenciada pelo aumento das doses de $\mathrm{Zn}$ em solução. Não houve diferença entre E. maculata e E. urophylla na concentração de $\mathrm{Mg}$ na parte aérea, e houve redução de $52 \%$ na dose de $1.600 \mu \mathrm{M}$ de $\mathrm{Zn}$, que permitiu atingir concentração mínima de $2,3 \mathrm{~g} \mathrm{~kg}^{-1}$, porém não atingiu níveis de deficiência para o crescimento das espécies. Nas raízes, houve também decréscimo na concentração de $\mathrm{Mg}$, sendo E. urophylla o que apresentou maior decréscimo na dose de $400 \mu \mathrm{M}$ de $\mathrm{Zn}$, que caracteriza a alta competitividade entre o $\mathrm{Zn}$ e $\mathrm{Mg}$, e que explica a redução na concentração de $\mathrm{Mg}$ na parte aérea, mostrando que houve participação de deficiência induzida de $\mathrm{Mg}$ nos sintomas de toxidez de $\mathrm{Zn}$, como resultado da similaridade do

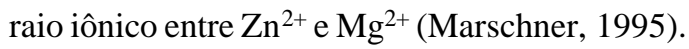

$\mathrm{O}$ aumento das doses de $\mathrm{Zn}$ exerceu efeitos significativos sobre a translocação de $\mathrm{P}, \mathrm{Ca}$ e $\mathrm{S}$ nas espécies de eucalipto (Figura 5). Houve incremento da translocação de $\mathrm{P}$, Ca e S em decorrência das doses de Zn. Esse comportamento pode ser atribuído ao efeito fitotóxico do $\mathrm{Zn}$ na perda de seletividade da membrana, causando influxo passivo destes nutrientes para a parte aérea. O incremento na translocação de $\mathrm{S}$ em resposta às doses de $\mathrm{Zn}$ pode estar relacio-

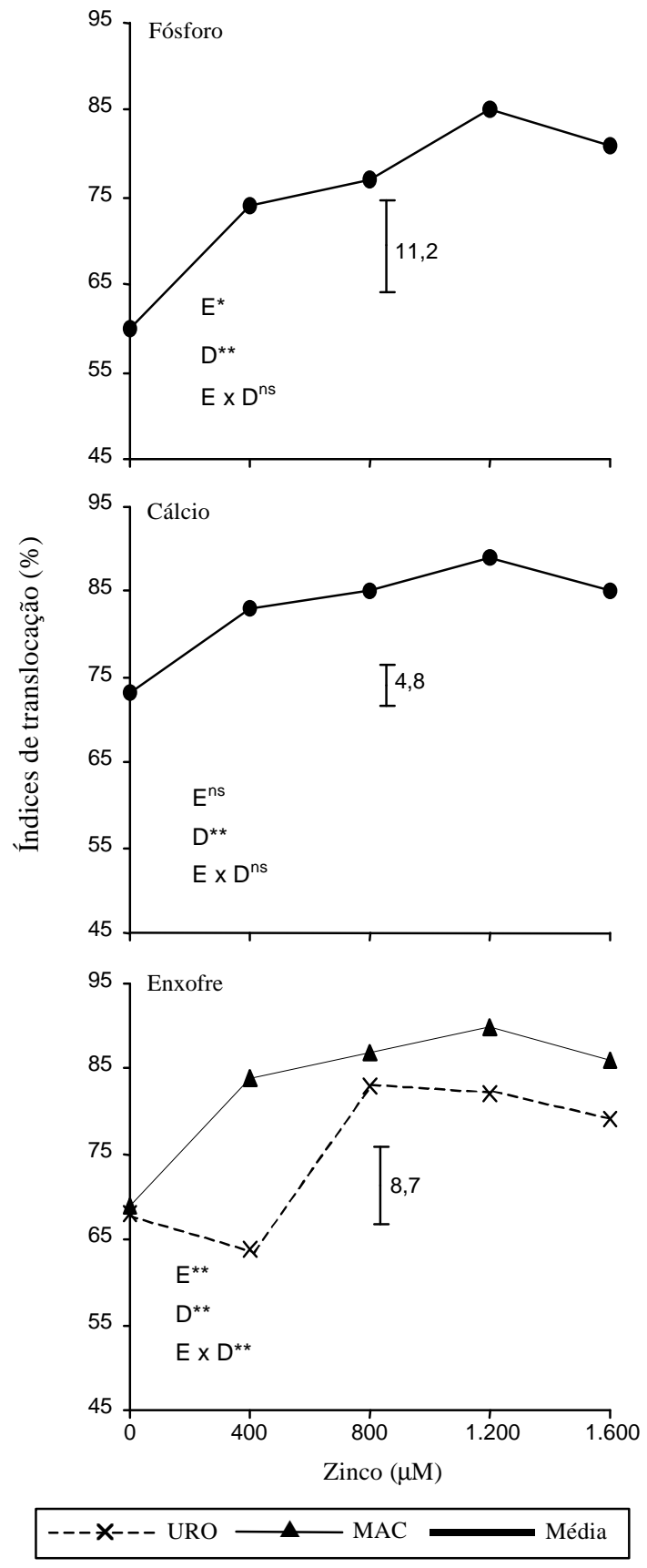

Figura 5. Índices de translocação (\%) de $\mathrm{P}, \mathrm{Ca}$ e $\mathrm{S}$ em duas espécies de eucalipto (MAC: E. maculata; URO: E. urophylla) crescidas em solução nutritiva com doses crescentes de Zn. E (espécie), D (dose de Zn) e interação E x D (ns: não-significativo; *p $\leq 0,05 ; * * \mathrm{p} \leq 0,01)$. A barra vertical representa a DMS (dose) do teste de Tukey a $5 \%$ de probabilidade. 
nado com mecanismos de tolerância das plantas ao excesso deste metal. Isto ocorre através da produção de substância intercelulares ricas em cisteína, ou seja, as fitoquelatinas (Fontes, 1992; Meharg, 1994; Keltjens \& Beusichem, 1998). Grill et al. (1987) afirmam que $\mathrm{Zn}^{2+}$ é um íon ativo a induzir a síntese desses compostos ricos em $\mathrm{S}$ e que estes têm a característica de complexar $\mathrm{Zn}$ nas plantas.

\section{Conclusões}

1. E. maculata e E. urophylla mostram-se sensíveis às doses crescentes de $\mathrm{Zn}$ em solução, exibindo sintomas de fitotoxidez, manifestados principalmente como clorose, escurecimento das raízes e inibição do crescimento das plantas.

2. E. maculata apresenta doses críticas na solução, e níveis críticos de toxidez, na planta, mais altos do que E. urophylla, indicando que E. maculata é mais tolerante do que E. urophylla.

3. Altas doses de $\mathrm{Zn}$ reduzem as concentrações de Fe e Ca na parte aérea a níveis considerados deficientes para o crescimento das duas espécies de eucalipto estudadas.

4. A translocação de Fe é altamente inibida pelo excesso de Zn, e esta deficiência induzida é uma das possíveis causas da fitotoxidez do Zn no eucalipto.

\section{Referências}

ABICHEQUER, A. D.; BOHNEN, H. Eficiência de absorção, translocação e utilização de fósforo por variedades de trigo. Revista Brasileira de Ciência do Solo, Campinas, v. 22, n. 1, p. 21-26, 1998.

AGARWALA, S. C.; BISHT, S. S.; SHARMA, C. P. Relative effectiveness of certain heavy metals in producing toxicity and symptoms of iron deficiency in barley. Canadian Journal of Botany, Ottawa, v. 55, p. 1299-1307, 1977.

BACCOUCH, S.; CHAOUI, A.; EL FERJANI, E. Nickel toxicity: effects on growth and metabolism of maize. Journal of Plant Nutrition, Monticello, v. 21, n. 3, p. 577-588, 1998.

BAKER A. J. M. Accumulators and excluders strategies in the response of plants to heavy metals. Journal of Plant Nutrition, Monticello, v. 3, n. 1/4, p. 643-654, 1981.
BARCELÓ, J.; POSCHENRIEDER, C. Respuestas de las plantas a la contaminación por metales pesados. Suelo y Planta, Madrid, v. 2, n. 2, p. 345-361, 1992.

BRECKLE, S. W.; KAHLE, H. Effects of toxic heavy metals $(\mathrm{Cd}, \mathrm{Pb})$ on growth and mineral nutrition of beech (Fagus sylvatica L.). Vegetatio, Dordrecht, v. 101, p. 43-53, 1992.

BROWN, S. L.; CHANEY, R. L.; ANGLE, J. S.; BAKER, A. J. M. Zinc and cadmium uptake by hyperaccumulator Thlaspi caerulescens grown in nutrient solution. Soil Science Society of America Journal, Madison, v. 59, p. 125-133, 1995.

CLARK, R. B. Characterization of phosphates in intact maize roots. Journal of Agricultural and Food Chemistry, Washington, v. 23, p. 458-460, 1975.

DAVIS, R. D.; BECKETT, P. H. T.; WOLLAN, E. Critical levels of twenty potentially toxic elements in young spring barley. Plant and Soil, Dordrecht, v. 49, p. 395-408, 1978.

DELL, B.; MALAJCZUK, N.; GROVE, T. S. Nutrient disorders in plantation eucalypts. Canberra : BPD Graphic, 1995. 104 p.

DELL, B.; ROBINSON, J. M. Symptoms of mineral nutrient deficiencies and the nutrient concentration ranges in seedlings of Eucalyptus maculata Hook. Plant and Soil, Dordrecht, v. 155/156, p. 255-261, 1993.

EUCLYDES, R. F. Manual de utilização do programa SAEG (sistema para análises estatísticas e genética). Viçosa : UFV, 1983. 59 p.

FONTES, R. L. F. Zinc toxicity in soybeans as affected by plant iron and sulfur. Raleigh : North Carolina State University, 1992. 138 p. Ph.D. Thesis.

FONTES, R. L. F.; COX, R. Iron deficiency and zinc toxicity in soybean grown in nutrient solution with different levels of sulfur. Journal of Plant Nutrition, New York, v. 21 , n. 8 , p. 1715-1722, 1998a.

FONTES, R. L. F.; COX, R. Zinc toxicity in soybean grown at high iron concentration in nutrient solution. Journal of Plant Nutrition, New York, v. 21, n. 8, p. 1723-1730, 1998b.

GRILL, E.; WINNACKER, E. L.; MEINHART, H. L. Phytochelatins, a class of heavy-metal-binding peptides from plants, are functionally analogous to metallothioneins. National Academy of Sciences of the United States of America Proceedings, Washington, v. 84, p. 439-443, 1987. 
HUANG, J. W.; CUNNINGHAM, S. D. Lead phytoextraction: species variation in lead uptake and translocation. New Phytologist, Cambridge, Grã-Bretanha, v. 134, p. 75-84, 1996.

KABATA-PENDIAS, A.; PENDIAS, H. Trace elements in soils and plants. Boca Raton : CRC, 1985. $315 \mathrm{p}$.

KELTJENS, W. G.; BEUSICHEM, M. L. van. Phytochelatins as biomarkers for heavy metal toxicity in maize: single metal effects of copper and cadmium. Journal of Plant Nutrition, New York, v. 21, n. 4, p. 635-648, 1998.

MALAVOLTA, E. Fertilizantes e seu impacto ambiental: micronutrientes e metais pesados, mitos, mistificações e fatos. São Paulo : ProduQuímica, 1994. 153 p.

MARQUES, T. C. L. L. de S. e M.; MOREIRA, F. M. de S.; SIQUEIRA, J. O. Crescimento e teor de metais de mudas de espécies arbóreas cultivadas em solo contaminado com metais pesados. Pesquisa Agropecuária Brasileira, Brasília, v. 35, n. 1, p. 121-132, jan. 2000.

MARSCHNER, $\mathrm{H}$. Mineral nutrition of higher plants. 2. ed. San Diego : Academic, 1995. 902 p.

MEHARG, A. A. Integrated tolerance mechanisms: constitutive and adaptive plant responses to elevated me- tal concentrations in the environment. Plant, Cell and Environmental, Oxford, v. 17, p. 989-993, 1994.

MOURA FILHO, G.; CRUZ, C. D. Fealc for Windows v.1.1: programa para cálculo do $\mathrm{F}$ corrigido em análises de regressão: teste dos coeficientes. Viçosa : UFV, 1996. Software.

POLLARD, A. J.; BAKER, A. J. M. Deterrence of herbivore by zinc hyperaccumulation in Thlaspi caerulescens (Brassicaceae). New Phytologist, Cambridge, Grã-Bretanha, v. 135, p. 655-658, 1997.

SALT, D. E.; PRINCE, R. C.; PICKERING, I. J.; RASKIN, I. Mechanisms of cadmium mobility and accumulation in Indian mustard. Plant Physiology, Rockville, v. 109, p. 1427-1433, 1995.

SAMARAKOON, A. B.; RAUSER, W. Carbohydrate level and photoassimilate export from leaves of Phaseolus vulgaris exposed to excess cobalt, nickel and zinc. Plant Physiology, Rockville, v. 63, n. 6, p. 1165-1169, 1979.

SHAW, A. J. Heavy metal tolerance in plants: evolutionary aspects. New York : CRC, 1989. 355 p.

WOOLHOUSE, H. W. Toxicity and tolerance in the responses of plant to metals. In: LANGE, O. L.; OSMOND, C. B.; ZIEGLER, H. (Ed.). Encyclopedia of plant physiology. Berlin : Springer, 1983. v. 12C, p. 245-300. 